\title{
Las cosas están vivas: relaciones entre cultura material, comunidades y legislación arqueológica
}

\section{These Things are Alive: Relationships Between Material Culture, Communities, and Archaeological Legislation}

\author{
Lucio Menezes \\ Departamento de Antropología y Arqueología \\ Universidad Federal de Pelotas. Rio Grande do Sul, Brasil \\ Investigador del CNPq \\ luciomenezes@uol.com.br; lucio.menezes@pq.cnpq.br
}

Recibido: $10-12-2014$

Aceptado:11-03-2015

\begin{abstract}
Resumen
Este artículo analiza las relaciones entre legislación arqueológica, cultura material y comunidades. Presenta un panorama histórico de las legislaciones sobre patrimonio cultural en el Occidente y sus usos contemporáneos. Posteriormente, discute la manera en que prácticas arqueológicas alternativas, como la Arqueología comunitaria, examinan dicho problema. Esta discusión general, que será hecha en la última parte del artículo, es muy relevante actualmente, especialmente en Brasil, país que se encuentra abrumado por la Arqueología empresarial.
\end{abstract}

Palabras Clave: legislación arqueológica, comunidades, cultura material, Arqueología empresarial.

\begin{abstract}
This paper deals with the relationship between different sets of archaeological legislation, material culture and communities. First it presents a historical sketch of the heritage legislation in the West and its contemporary uses. Secondly, it shows how alternative archaeological agencies, such as community archaeology, deal with these problems. The discussion is especially relevant in Brazil, where contract archaeology is presently overwhelming, and the issue is raised in the last part of the paper.
\end{abstract}

KEY worDs: Archaeological Legislation, Communities, Material Culture, Contract Archaeology. 
"Lo que une a los individuos no es cemento" (Norbert Elias. La sociedad de los individuos, 1939)

\section{Introducción}

La relación entre cultura material y comunidades permite múltiples aproximaciones, a partir de áreas disciplinares muy diversas. Una de las cuestiones centrales, puntualizada bajo diferentes matices en el libro organizado por el antropólogo Arjun Appadurai (1988), se refiere a cómo las cosas tienen vida social y se enraízan en las cosmologías de las comunidades. Los estudios sobre materialidad discuten los modos en que dichas cosas y sociedades se co-producen y cómo la cultura material sirvió y aún sirve, como punto de apoyo para el gobierno, nacional o colonial, de las comunidades (Buchli 2007; Gosden 2004: Thomas 1991; Given 2004). Otra aproximación significativa es la del sociólogo Bruno Latour. Rompiendo el dualismo sujeto-objeto, propone una interpretación simétrica entre humanos y no humanos y además enfatiza la acción social de las cosas sobre ambos (Latour 2008). En resumen, en los últimos años descubrimos que la cultura material está animada. Está viva y palpita. Las cosas tienen alma, están estructuradas e influyen en nuestros comportamientos (Gosden 2005). Circunscriben memorias sociales, fijan calendarios, inspiran celebraciones (Jones 2007). Comunidades y cosas se encuentran enredadas (Olsen 2010; Hodder 2012).

Sin embargo, hay una clase de cosas que no pertenece plenamente a las comunidades. Continúa ejerciendo acción social sobre ellas, pero no les pertenece completamente. Es decir, las cosas están ahí y las comunidades les dan uso, mas reina sobre ellas una ambivalencia fundamental. Las cosas están en el lugar. Todos las ven. Las comunidades pueden tocarlas. Pueden darles significados, asociarlas afectivamente con la memoria ancestral, convertirlas en marcos del derecho a la posesión de la tierra y, por consiguiente, de la lucha por la ciudadanía. No obstante, incluso estando en el lugar, esa clase de cosas gravita en lo global, en la categoría difusa de lo universal, de lo que pertenece a la nación o a toda la humanidad.

Me refiero con esto a la clase de cosas que denominamos arqueológicas. Recaen sobre ellas sanciones legislativas variadas, que abarcan desde los niveles municipales hasta los nacionales e internacionales. Generalmente, cosas arqueológicas y comunidades se incluyen en la retórica pedagógica de los nacionalismos, la cual disemina a la Nación entre cada uno de sus miembros (DissemiNation, conforme el creativo neologismo de Homi Bhabha 1990). Ambas están atadas por el ligamento pegajoso de la "comunidad imaginada" (Andersen 1983): ningún individuo de una nación, por pequeña que ella sea, conoce todas las cosas arqueológicas con las que se compone su identidad nacional.

Las legislaciones arqueológicas tienen el poder de nombrar las cosas que definen identidades nacionales, a despecho, casi siempre, de los deseos y deliberaciones de las comunidades locales. Aquel sitio y todas sus cosas están ahí, en la comunidad; la comunidad puede pisarlo, manipular las cosas que forman el "registro arqueológico"; usarlo como fuente de recurso alimenticio o como materialización de lo sagrado (Fourmile 1989). Sin embargo, la legislación, del lado de los arqueólogos y arqueólogas, puede otorgarles valor nacional o universal. Y, por más que tanto arqueólogas como arqueólogos puedan consultar a las comunidades y considerar sus demandas, la legislación, como expresión de poder de soberanía de una nación o del estatuto de lo universal, dice a las comunidades que, en efecto, aquellas cosas están allí, aunque pareciera que no estuviesen. No son, en definitiva, de nadie, sino de todos los que viven en una nación o de toda la humanidad; pertenecen a todos "nosotros", incluso a aquellos que nunca las vieron y jamás las verán.

Los estudios contemporáneos sobre cultura material, con sus enunciados acerca de la vida social y estructurante de las cosas, raramente se detienen en esa ambivalencia fundamental de las legislaciones arqueológicas. Es habitual que estos estudios de cultura material analicen las cosas como significantes; disciernan sobre sus re-semantizaciones, siguiendo los significados cambiantes que adquieren en sus desplazamientos por diferentes contextos institucionales, culturales y políticos. Describen la larga biografía de las cosas: su renacimiento durante las excavaciones (hasta entonces estaban enterradas y muertas), su trayectoria en laboratorios, su afiliación a un taxón y su disposición en una colección, hasta su posterior aprisionamiento en las vitrinas de los museos.

En este sentido, el primer cautiverio de las cosas lo decretan las legislaciones arqueológicas. Ellas se adueñan de las cosas, modelándolas en una suerte de categorías nacionales y universales. 
Es cierto, igualmente, que las comunidades presionan a legisladores, arqueólogos y gestores del patrimonio cultural para descentralizar la gestión, la salvaguardia y el uso de la cultura material; se puede indicar, en las últimas décadas, una serie de cambios en las legislaciones patrimoniales y códigos de ética de investigación arqueológica gracias a la acción combativa de movimientos civiles (Schofield y Johnson 2006). A pesar de esto, tal como argumentaré más adelante, todas estas transformaciones han acabado cooptadas en favor, principalmente, de la mercantilización de la cultura material, de la vía libre para obras de ingeniería y el turismo globalizado. La Arqueología empresarial, aquí, tiene un papel bastante marcado: es una "realidad de transacción" (Foucault 2008) entre las cosas y el mundo liberal, una estrategia en los juegos de poder que permiten la neo-colonización de las cosas por medio del mercado. Esta arqueología posibilita que el mercado, a su vez, se justifique mediante el lenguaje patrimonial. De modo que hoy, las cosas arqueológicas aparecen revestidas no sólo por las tintas del nacionalismo o por los colores del estatuto de lo universal; son, también, fuentes de legitimación del mercado.

Por esta razón, mí objetivo en este artículo es discutir el tema de las relaciones entre cultura material, comunidades y legislaciones arqueológicas. Inicialmente, haré un esbozo histórico de las legislaciones sobre patrimonio cultural en Occidente y detallaré sus efectos contemporáneos; luego describiré la forma en la que determinadas prácticas arqueológicas, por ejemplo la Arqueología comunitaria, lidian con dicha problemática. Toda esta discusión es bastante pertinente para el momento actual, especialmente en el caso brasileño, que se encuentra abrumado por la Arqueología empresarial. Trataré este caso en la última parte del capítulo, a modo de conclusión.

\section{Las cosas en las redes de la legislación arqueológica}

La relación entre cosas y legislación arqueológica en Occidente tiene una larga historia de genealogías y apropiaciones articuladas con el proceso de formación de la idea de patrimonio cultural. Cuando emergió en la Modernidad, el patrimonio cultural fue, sobre todo, creación de las instituciones jurídicas del Estado. Parafraseando al antropólogo Victor Turner (2005), el patrimonio cultural surgió como un momento de paso: al des- integrar las comunidades tradicionales el Estado moderno inventó el patrimonio cultural, proscribiendo o adueñándose de los símbolos antiguos. Los métodos sangrientos de creación de los Estados modernos, a partir del Renacimiento, fueron acompañados por la definición del buen gusto, de la elección de los estilos que debían ser transmitidos: el reconocimiento de un canon de maestros y la delimitación de un corpus artístico que debía preservarse. Se instituyeron, sobre todo en los nacientes Estados de la península itálica, legislaciones de protección y conservación del patrimonio. Una de las más célebres es el decreto de 1601, por medio del cual el gran duque Fernando de Médici promulgó una lista de dieciocho pintores del pasado cuyas obras no podrían venderse en el exterior (Gombrich 1992).

Se inició así en Occidente, el proceso histórico que transformó al patrimonio en una categoría de pensamiento y acción social. Al elegir un canon, se concebía una manera de comprender el mundo y actuar en él. De ahí la mezcla entre Estado y canon, entre las formas jurídicas de gobernabilidad y el arte. Se pensaba y se constituía el Estado no sólo por la fuerza del Derecho y la conquista, sino también, como obra de arte, resultado de la creación consciente basada en manifiestos y técnicas (Burckhardt 2012). Así, el vocabulario político de la Modernidad se codificó, desde su inicio, en prescripciones sobre los usos del pasado (Skinner 1996). Se pasó a seleccionar e institucionalizar la herencia que los muertos legaron a los vivos (¡una de las manifestaciones filosóficas de esto es el espanto de Comte con la superpoblación de los cementerios!).

En Occidente las sociedades del presente dispusieron las reglas del culto al pasado (todas las sociedades, bien en forma de mito o bien en forma de historia, crean sus reglas de culto al pasado). El estudio del pasado, inicialmente por medio de la Filología, la Arqueología y la Historia (por lo menos desde Vico), se vuelve epistemológico, índice de la organización política del presente y germen de la imaginación utópica, de las proyecciones futuras de la sociedad (Horkheimer 1970). Dicho giro epistemológico se radicalizó en el siglo XVIII, el "siglo de las revoluciones": se interroga la actualidad a través del filtro del pasado, del que se seleccionan porciones para circunscribir la singularidad de una trayectoria histórica, un "nosotros" que remitiría a una configuración cultural singular (Foucault 1996).

Se acabó entendiendo que la noción de ciudadanía se encontraba atada inquebrantablemente a 
la Historia, a las cosas, al territorio y a la lengua. Es decir, desde el siglo XVIII, en un proceso que no hizo más que acentuarse progresivamente a partir del siglo XIX, el individuo, el ciudadano del mundo liberal, se volvió impensable fuera de la red social que lo liga a las tradiciones nacionales dictadas por el Estado (Elias 1994). De ahí la proliferación de leyes que aseguran un destino específico a las cosas arqueológicas: estas cosas se restauran, preservan, exhiben en los museos y en las plazas públicas. La legislación patrimonial como praxis en la constitución histórica moderna de la jurisprudencia - se apoya, de este modo, en un conjunto de conocimientos expertos y ciencias: se convoca a museólogos, antropólogos, arqueólogos, historiadores, conservadores y arquitectos para sancionar el veredicto patrimonial.

Nada de esto nos resulta extraño. En nuestras sociedades, las prácticas jurídicas, en colaboración con varias ciencias, instituyeron la indagación y el examen como forma de llegar a la verdad y crear una técnica de gestión, una modalidad de administración estatal de la economía y de la política (Foucault 2002). Lo que llamamos patrimonio cultural no escapó a esos procedimientos del examen y la indagación: se recurrió a las ciencias para fundar una razón jurídica sobre el pasado, administrar el legado cultural y decir a los individuos cuáles son, y cuáles no, sus filiaciones identitarias. En nuestras sociedades existe, aún hoy, un estatuto jurídico de las identidades sociales, para cuyo funcionamiento se activa la patrimonialización de las cosas. Para decirlo con un juego de palabras: desde el siglo XVIII, ningún gobierno gobierna sin patrimonio cultural (Ferreira 2009a).

De modo que, pasada más de una década de siglo XXI, disponemos de una abundancia legislativa que regula las cosas arqueológicas. Internamente, en Brasil, tenemos los "Planos Directores", vinculados a las Secretarías de Cultura de los municipios; asimismo, a nivel federal, las resoluciones del Instituto del Patrimonio Histórico y Artístico Nacional, concretamente la Ordenanza $n^{\circ} .230$ (de 17 de diciembre de 2002), que regula las fases de obtención de permisos ambientales para las obras de ingeniería que impactan o destruyen el patrimonio arqueológico; finalmente, en la esfera transnacional, tenemos la actuación, entre otros organismos, de la UNESCO y, también, varias Cartas Internacionales, emanadas de convenciones a partir de las cuales se publican documentos que guían los procedimientos técnicos y éticos para la investigación, conservación y protección de las cosas arqueológicas.
De ahí que las cosas arqueológicas se vean cercadas por la retórica legislativa de preservación. Pero, ¿cuál es realmente el tono que caracteriza esta imaginación legislativa? Tomemos el ejemplo de la UNESCO. El lenguaje patrimonial de la institución, pese a la incorporación de la noción de diversidad cultural, refuerza claramente valores predominantemente occidentales. Eso se observa en la idea de herencia cultural mundial y universal: reconfigura y se apropia de antiguos proyectos coloniales al conformar territorios delimitados por fronteras nacionales (Meskell 2002). Es innegable, sin embargo, que muchas comunidades, fortalecidas mediante movimientos civiles, han impulsado la reformulación de las legislaciones arqueológicas. Es el caso, por ejemplo, de los aborígenes australianos y de los indígenas norteamericanos, quienes, junto a arqueólogas y arqueólogos, garantizan el derecho de gestión de su propio patrimonio y de repatriación arqueológica (Simpson 2001; Colley 2002; Ferreira 2009a). De modo que podemos afirmar que sin los movimientos civiles no habría crítica al canon occidental y al logocentrismo de la retórica patrimonial, las legislaciones arqueológicas y los códigos de ética de investigación permanecerían estancados y varias disciplinas, como la Arqueología de la esclavitud, no habrían emergido (Ferreira 2009b).

Partiendo de esto, me pregunto si a estas alturas de los acontecimientos mundiales, el mercado y las prácticas nacionalistas no se están apropiando constantemente de dichas legislaciones y códigos de ética. Cosas y monumentos, en varias regiones del mundo, se ven progresivamente atrapadas en las redes de los "nuevos" y "viejos" nacionalismos, tanto en el Este de Europa como en el Oriente Medio, Japón y China (Kohl et al 2007). En esto proceso, incluso el discurso del multiculturalismo resulta apetecible, especialmente en América Latina. Aunque las diversas legislaciones arqueológicas, modelos de gestión del patrimonio e, incluso, las constituciones modernas, incorporen el concepto de multiculturalismo, lo hacen para homogeneizar las diferencias e impedirles el diálogo; lo evocan para recrear narrativas nacionalistas y colonialistas (Guthrie 2010; Armstrong-Fumero 2009; Gnecco 2009). El problema es que nuestras sociedades, incluso admitiendo y celebrando sus culturas plurales, no han abandonado la "tiranía burocrática", con su principio de autoridad centralizada en la Ley para regir a los individuos y sus identidades (De Certeau 1993). 
Al encargarse de las cosas, las legislaciones arqueológicas y su reciente apelativo multicultural, parecen haber confeccionado "mordazas sonoras" (Sartre 1979) para grupos subalternos. Las legislaciones arqueológicas han sido hábiles para actuar como aparatos de captura (Deleuze y Guattari 1980), absorbiendo el habla de los grupos subalternos para burocratizarla y darle un pretendido tono democrático. Conjugan los verbos del reparto de las cosas, de su gestión comunitaria, pero solamente para administrarlas mejor y retener la diversidad cultural que ellas componen. ¿Dónde resultan más visibles esas artimañas? Precisamente, en la exégesis de los juristas ingleses del patrimonio, aquellos que, en la línea de John Henry Merryman (2000), John Carman (2005) y Derek Gillman (2010), tratan el patrimonio arqueológico como herencia de toda la humanidad, como "recurso cultural" que debe administrarse en foros internacionales y explotarse como dividendo turístico. Vacían así la pluralidad de representaciones que las comunidades confieren a las cosas, amalgamándolas en solo concepto. Es significativo que esta vertiente jurídica de análisis se incluya en los intereses del mercado turístico: lo que interesa del turismo nacional e internacional que alimenta tarjetas de crédito, redes de hoteles y compañías aéreas, es, obviamente, el consumo. Y el pretexto del consumo es el disfrute del llamado patrimonio universal.

La relación entre Turismo y Arqueología surgió a lo largo de los siglos XIX y XX, junto con la industrialización, el colonialismo y la construcción de identidades nacionales (Chambers 2000). Sin embargo, desde finales del siglo XX, la importancia del turismo arqueológico (es obvio que no sólo el arqueológico) ha aumentado considerablemente como industria global, convirtiéndose en una excusa para el desarrollo económico en todo el mundo. Se reitera en este proceso, lo que ya se había hecho en el siglo XIX: el uso y abuso de las cosas arqueológicas para estructurar identidades nacionales y ahora neocoloniales. Se introdujo, entretanto, una innovación: la participación de las comunidades locales en las excavaciones arqueológicas y en la gestión del patrimonio (Baram 2007). La mercantilización de las cosas arqueológicas para el turismo, aparte de reinscribir las identidades nacionales en los nuevos registros proporcionados por los viajes internacionales, acomoda confortablemente las interpretaciones y cosmologías de las comunidades. Como Silverman (2002) demuestra para el caso del Perú, la participación de las comunida- des, invocadas bajo el signo de la multivocalidad y del multiculturalismo, asegura la autenticidad de la industria turística.

En el contexto en que trabajo, América del Sur, las cosas arqueológicas, como espectáculos del turismo global, se conjugan con los procesos de expropiación de tierras para favorecer hoteles, multinacionales y redes de servicios (Belli y Slavutsky 2010; Rivolta et al. 2010). Las comunidades se quedan, casi siempre, con las sobras de la economía turística; la venta de artesanías y empleos subalternos, pero nunca con la gestión comunitaria de los negocios y, tampoco con la dirección efectiva de los llamados proyectos de desarrollo auto-sostenible. Se reconfiguran, de ese modo, las herencias del colonialismo en sus aspectos más evidentes: la apropiación de las historias nativas y la expropiación de tierras.

El turismo arqueológico está marcado, además, por una paradoja. El turista se sumerge en la ambivalencia de las políticas de representación y gestión del patrimonio cultural. Por un lado, se encuentra sometido a las retóricas locales del patrimonio arqueológico, con sus nociones de identidad regional y nacional. Está sometido, por lo tanto, a la idea de patrimonio como lugar, es decir, a la identidad en tanto que sinónimo de territorio compartido, aunque, para el gusto del turista, tal identidad pueda tener un toque exótico (y, para las industrias turísticas, mucho mejor es que lo tenga). Por otro lado, siguiendo los enunciados del antropólogo Marc Augé (2007), el turismo arqueológico, con su retórica de lo universal, de la identidad compartida por las realizaciones culturales de toda la humanidad, se articula como no-lugar. Como en los supermercados, terminales, centros comerciales y aeropuertos, el turismo arqueológico intenta uniformizar prácticas culturales. Al turista se le presenta lo mismo, lo reconocible, y el turismo arqueológico se despliega como espectáculo de consumo del mundo globalizado.

\section{Esbozando el problema}

Las redes de las prácticas nacionalistas y del turismo global acaban así atrapando las cosas. Dicho proceso se incrementó a partir de los años 90, con el avance del neoliberalismo y el recrudecimiento de las guerras a escala global. No es casualidad que al final de los años 90 una obra apuntase a que la arqueología estaba "bajo el fuego" (Meskell 1998). No obstante, las comunidades están 
combatiendo el uso de las cosas arqueológicas como alegoría nacionalista y mercantilización. Cada vez más, en las sociedades contemporáneas, las comunidades se embarcan en movimientos de descolonización de la Arqueología en sus territorios (Smith y Wobst 2005). De modo que en las últimas dos décadas, han ido emergiendo áreas disciplinares que reconfiguran el trabajo arqueológico junto a las comunidades. En general, se conceptualiza como Arqueología pública (Merriman 2004), Arqueología colaborativa (ColwellChanthaphonh y Ferguson 2008; Macdavid 2004) y Arqueología comunitaria (Marshall 2002; Tully 2007). Se entienden como democratización del conocimiento arqueológico (Shackel 2001; Holtorf 2006) y una "arqueología vista desde abajo" (archaeology from below) (Faulkner 2000); se describen, además, como teorizaciones novedosas sobre las relaciones entre presente y pasado, la investigación arqueológica y el público (Simpson y William 2008).

No hay una ontología propiamente dicha que una las propiedades de estos campos. Pero se puede hablar de dos elementos metodológicos comunes, muy presentes en la bibliografía. El primero es la etnografía arqueológica. Son tres los objetivos de la etnografía arqueológica: entender las cosmologías y representaciones de las comunidades sobre las cosas arqueológicas y sus narrativas sobre el pasado; analizar las políticas de negociación de identidades y los conflictos que caracterizan a las comunidades; y promover un espacio intercultural, de diálogo permanente entre el equipo arqueológico y la comunidad, abriéndose así oportunidades para la educación mutua y también para el establecimiento de comparaciones entre los discursos arqueológicos y locales (Hamilakis y Anagnostopoulos 2009).

No se trata, entonces, de educación patrimonial, del modo como esta viene siendo realizada sobre todo en Brasil (Ferreira 2010a; Ferreira y Sanches 2011). En la etnografía arqueológica, al primero que se debe educar es al arqueólogo mismo. Se busca, pues, crear un contrapeso en la asimetría de poder entre los arqueólogos y las comunidades. El arqueólogo no educa unilateralmente a la comunidad, sino que aprende con ella: en el trabajo etnográfico, dialoga acerca de las búsquedas identitárias de la comunidad; descubre (descubrir en la doble acepción de inventariar e inventar) la pluralidad de significados que las comunidades atribuyen a los artefactos y sitios arqueológicos. La etnografía arqueológica, vista así, tiene fundamento simultáneamente político y epistemológico. Lleva a pensar la diversidad cultural como una relación inseparable entre el presente y el pasado y, también, a considerar las tecnologías de gobierno promovidas por la activación del patrimonio cultural.

El segundo elemento metodológico común en estos temas arqueológicos es la integración de las comunidades en todas las etapas de investigación arqueológica: desde la formulación del proyecto de investigación, elaborado en consonancia con las demandas de las comunidades, hasta la proyección, exploración, excavación de sitios arqueológicos, conservación y análisis de la cultura material en el laboratorio. Se trata de dotar a las comunidades con instrumentos útiles para el manejo de su patrimonio arqueológico. Las políticas de representación del patrimonio arqueológico, sus formas de difusión, exploración y administración, son decididas, conjuntamente, entre las comunidades y los arqueólogos. Las comunidades, en este sentido, ya no tienen la función de "informantes", guías locales para la búsqueda de sitios o "peones" en las excavaciones. Lo que se pretende es nivelar las relaciones de poder entre arqueólogos y comunidades y descentralizar la autoridad de la institución arqueológica.

El arqueólogo se convierte en colaborador de la comunidad. Busca disfumar, en este cuadro metodológico, su imagen tradicional: el extranjero, aliado de la administración colonial o del Estado nacional, que se apropia de las cosas arqueológicas locales y define, de forma monolítica, la "diversidad cultural de la humanidad". Al contrario, aquí, como en cualquier otra reflexión antropológica, la cosmología del arqueólogo se contrasta con la cosmología de la comunidad; el arqueólogo, en este punto, no sólo se re-inventa culturalmente, sino que descubre la pluralidad de significados que poseen los artefactos, los monumentos y el paisaje.

\section{De vuelta a las redes}

Esos campos arqueológicos, por lo tanto, apuntan hacia la gestión e interpretación compartida de las cosas arqueológicas y hacia la promoción de la diversidad cultural. Sin embargo, quizás, estas prácticas están hoy en día simplemente comenzando a esbozar el problema, al menos en la mayor parte de los casos. Como ya señalé, las legislaciones arqueológicas continúan capturando las cosas en sus redes y salvaguardando 
el nacionalismo y el turismo global (Appadurai 2002). Al apuntar esto no estoy afirmando que el trabajo arqueológico con las comunidades, bien como la activación de las diversas legislaciones arqueológicas, sea parte de un plan maquiavélico, sobre el cual existe un acuerdo consensuado por parte de élites, gobiernos y el mercado turístico global. Tampoco estoy defendiendo que las arqueólogas y los arqueólogos sean agentes acríticos e ingenuos ante la mercantilización, nacionalización y colonización de las cosas arqueológicas. Finalmente, no estoy minimizando la actuación incisiva de los movimientos civiles que han promovido todas las transformaciones que, al menos desde los años 60, tuvieron lugar en las legislaciones arqueológicas, en los códigos de ética de las investigaciones, la instauración del punto de vista nativo en la Historia y la descolonización metodológica de la Arqueología y de la Antropología, entre otras transformaciones cruciales y decisivas.

No obstante, me parece que existe hoy una acentuada tendencia a que el trabajo arqueológico con las comunidades o la Arqueología pública (terminología bajo la cual se engloban, equivocadamente, diferentes disciplinas arqueológicas, como Arqueología comunitaria y la Arqueología colaborativa), no se opongan claramente al nacionalismo, el colonialismo o el mercado. De nuevo aclaro mi argumento de modo antitético. Sin lugar a dudas, la Arqueología puede traer beneficios públicos (Little 2002). Y, en efecto, se trata de un "giro epistemológico" cuando, como si fuera en el teatro de Augusto Boal, el público se transforma en poeta y protagonista de la obra, y la mano de obra anónima que empuña los instrumentos de excavación no es únicamente negra (Sheperd 2009). En Brasil, por ejemplo, dar protagonismo a las comunidades y visibilizar la institucionalización de proyectos de Arqueología colaborativa sería fundamental ante el actual contexto de lucha de las comunidades indígenas y afro-descendientes por la tierra. Lamentablemente, son pocos, en Brasil, los trabajos enfocados en esa línea (Cf. p. ej: Silva 2011; Eremites de Oliveira y Pereira 2012; Bezerra 2012; Funari y Bezerra 2012; Funari y Carvalho 2012), mientras latifundistas, vinculados o no a los escaños del Congreso Nacional brasileño, se organizan (cuando no están actuando violentamente) contra la demarcación de tierras indígenas y de afro-descendientes, tal como vemos cotidianamente en las ciudades de Brasil.
Se observa entonces, en este país y en otros lugares, que las cosas arqueológicas bajo el beneplácito y el ordenamiento de la legislación patrimonial, legitiman los llamados planes de crecimiento económico o proyectos de desarro1lo. La práctica contemporánea que engendra tal legitimación es la Arqueología empresarial que surgió, históricamente, como realidad de transacción entre las cosas arqueológicas y el liberalismo. Y hoy la Arqueología empresarial viene haciendo un uso muy particular de la Arqueología pública. Se vale de la disciplina, en una clara distorsión de su epistemología y objetivos, como estrategia de marketing, como publicidad. Los proyectos de desarrollo pueden destruir los sitios, pero la Arqueología empresarial, aliada a esa versión furtiva de la Arqueología pública, nos dice: "¡Aquí estamos nosotros para preservar el pasado para el futuro y educar a la comunidad!". De este modo las empresas y gobiernos conjugan ahora destrucción desarrollista con preservación del pasado. Cuando no actúan cínicamente, viendo a la Arqueología como algo que se debe tolerar, o pura y simplemente como un obstáculo para el calendario de realización de las obras (¡no se puede parar la obra por culpa de estas lascas!). Pero la tónica general, con rarísimas excepciones, es la que dictamina la propaganda.

Supongo que todos hemos visto, en nuestro propios países o en el exterior, folletos de propaganda (cuando no artículos y congresos científicos) donde se presenta a la Arqueología empresarial como Arqueología pública. En Brasil, especialmente, lo que se solía llamar educación patrimonial, ahora se acostumbra a clasificar como Arqueología pública, como si ésta se limitase a la pedagogía patrimonial. Detengámonos en los códigos de dicho marketing académico. En primer lugar, en los manuales, cartillas, folletos y propuestas de "Arqueología pública" se colocan los logos de las empresas e instituciones gubernamentales que pagaron por el trabajo arqueológico. Es algo similar a la propaganda de las empresas "ecológicas" de cosméticos, como Natura: explotemos ampliamente los recursos naturales, pues, al final, "trabajamos por la conciencia de sostenibilidad y tenemos, actualmente, veinte proyectos para mejorar la educación brasileña" (Cf. el sitio Natura Ekos e Instituto Natura). Los logos de la propaganda ilustran, en segundo lugar, la empresa de Arqueología empresarial que ganó la licitación para ejecutar el trabajo. Se vende el producto, muchas veces, exhibiendo orgullosamente fotos que representan la "ciencia en acción", donde 
los principios metodológicos de la Arqueología comunitaria son puestos en la escena: se muestra a la comunidad trabajando en todas las etapas de la investigación arqueológica, pero la realidad es que no se consulta previamente a la comunidad a la hora de elaborar el "proyecto de investigación" y tampoco se delibera con ella sobre los impactos económicos y ambientales que dichas obras de ingeniería podrán ocasionar. En resumen, se continúa tranquilamente con la "mejor" tradición autocrática del Estado brasileño: la imposición de proyectos sin consultar a la sociedad civil.

Leyendas y textos de las fotos hablan de despertar vocaciones arqueológicas y patrimoniales, en la garantía del derecho a la cultura y a la memoria ( como si las comunidades no tuviesen cultura y memoria!), en la educación de jóvenes y adultos, en el "empoderamiento", en formar a los profesores locales en los temas arqueológicos (casi siempre reducidos a la Prehistoria), en fomentar la artesanía local con motivos arqueológicos, y así sucesivamente. De lo que no se habla es de que los miembros de la comunidad vuelven a ser los antiguos "peones" de los trabajos arqueológicos. Tal vez en una escala todavía más explotadora, porque las comunidades, casi siempre, solo reciben dietas, y no disfrutan de los derechos comunes de los trabajadores en Brasil: vacaciones remuneradas, pagas extraordinarias, etc. Es casi el don del contacto colonial: "deme sus cosas y paisajes, le doy a cambio educación, cultura arqueológica, re-significación de su memoria y desarrollo". Así, la ironía del arqueólogo social latinoamericano Felipe Bate sobre la Arqueología pública es perfectamente plausible: se la puede entender como un discurso "altamente progresista y democrático, sumado a un paternalismo conmovedor" (Bate 1998: 12).

Como ya apuntó Chris Gosden (2001), la Arqueología se institucionalizó, en la Modernidad, como producto de la Filosofía liberal. Y no nos hemos liberado completamente de ese proceso. Eso es evidente en la propia aparición, en los Estados Unidos, de la conjugación entre Arqueología empresarial y Arqueología pública. El término Arqueología pública aparece acuñado, por primera vez, en la obra homónima de Charles McGimsey (1972). Lo que lo llevó a escribirla fue la legislación patrimonial que surgió en los Estados Unidos en los años 60, designada como Cultural Resource Management (CRM). McGimsey estaba preocupado, como tantos otros arqueólogos y arqueólogas, por la destrucción acelerada de sitios arqueológicos, llevada a cabo por los proyectos capitalistas de desarrollo. Sin embargo, en esa preocupación no descansa solamente la buena conciencia romántica de preservación o el llamado público ilustrado para la conservación de los "recursos culturales". Al contrario. Rápidamente la CRM y la Arqueología pública se colocaron al servicio de las empresas capitalistas y del gobierno, viabilizando obras de ingeniería y negocios turísticos. Aquí nació, bien nutrida y vigorosa, la Arqueología empresarial y su alianza con la Arqueología pública.

Ciertamente, la Arqueología procesual, que emergió en ese mismo contexto, se benefició enormemente con recursos destinados para la investigación. No obstante, tal como recientemente argumentó Patty Jo Watson (2008), una de las legítimas representantes de la Arqueología procesual, más del 90\% de la Arqueología practicada en los Estados Unidos hoy se hace por contrato, vinculándose a la CRM y a la Arqueología pública. Trigger (1984), en un artículo clásico, argumentaba, a mediados de los años 1980, que el procesualismo, con su búsqueda de regularidades transculturales y sujeción de las culturas a los sistemas, era expresión del imperialismo y nacionalismo de los Estados Unidos. El núcleo de la argumentación de Trigger es que la Arqueología se ubica en el centro del sistema mundial, en la acepción de Immanuel Wallerstein. Equivale esto a decir que durante la guerra fría, una parte de la Arqueología mundial se estaba institucionalizando dentro de la esfera del liberalismo.

Así pues, ese vínculo contemporáneo entre Arqueología empresarial y "Arqueología pública" hace que se identifiquen íntimamente con el mercado, reproduciendo desigualdades sociales con retóricas patrimoniales. La cuestión no es sólo permitir las obras desarrollistas, en la medida en que es fundamental para el funcionamiento del mercado. Se trata, igualmente, de acoger a las comunidades y todas sus cosas arqueológicas, cual piezas publicitarias. Se trata, aún más, de trasmitir la imagen de que la destrucción también construye. Destruye, pero preserva la pluralidad cultural. Devasta positivamente, inventariando paisajes, costumbres y cosas en los archivos, donde se podrán realizar posteriormente consultas sobre la diversidad que nos caracteriza históricamente. En esos archivos, el pasado estará registrado para beneficio del futuro.

Es este punto, las cosas arqueológicas se mercantilizan y actúan en el mundo liberal. Se enredan en las tramas del discurso liberal acerca de la pluralidad cultural, el cual, desde 1989, usurpa las 
diferencias y la diversidad con su idea de individuo y libertad (Bauman 1998). Aun hay más. Los lazos entre Arqueología empresarial y "Arqueología pública" rejuvenecen viejos nacionalismos y colonialismos. En este sentido, me gustaría narrar una experiencia personal. En mayo de 2012 tuve la oportunidad de participar, como invitado, en el congreso Archéologie de l'esclavage colonial, en el Musée du quai Branly, en París. Dicho congreso lo organizó el Institut National de Recherches Archéologiques Préventives (INRAP), vinculado al Ministerio de la Cultura y Comunicación de Francia. Ese instituto es responsable de la Arqueología empresarial que se hace en dicho país.

El INRAP, por lo tanto, centraliza, a través del Estado, los trabajos en Arqueología empresarial. Se refiere a una forma de gestión y ejecución de Arqueología empresarial muy distinta a lo que se ha denominado el "modelo brasileño" (para otros ejemplos, Cf. Ferreira y Sanches 2011), muy cercano al del "hombre cordial" descrito por Sérgio Buarque de Holanda, esto es, propenso a no discernir y a mezclar las áreas de actuación de lo público y de lo privado. Pues bien, me sorprendió poder constatar, en los folletos de propaganda del INRAP, tres eslóganes. El primero: nosotros excavamos su historia; entiendo que, ante tal evidencia, no se necesitan mayores comentarios sobre el fondo nacionalista de dicho slogan. El segundo se relacionaba con la Guinea francesa, cuyo tono era más o menos la siguiente: descubrimientos del pasado francés en Guinea. El fondo colonialista de este eslogan es también bastante evidente, pero me atrevo a decir que, en esta época, cuando tantos arqueólogos y arqueólogas se esmeran en refinar las aproximaciones postcoloniales y hablan de descolonización de la Arqueología, no deja de ser tremendo leer una frase tan colonialista como esta.

El tercer eslogan, a su vez, es bastante recurrente en la Arqueología empresarial que se hace fuera. Decía algo de este género: el creciente número de sitios excavados por el INRAP en Francia. Acompañaban dicho slogan datos cuantitativos y se representaban, en mapas de distribución, el número vertiginoso de sitios excavados por el INRAP. Moraleja de la historia: antes de la Arqueología empresarial, antes del INRAP, no conocíamos ese número de sitios arqueológicos. Cabe preguntarnos si los datos numéricos sobre la cantidad creciente de sitios arqueológicos registrados y excavados redundan, verdaderamente, en conocimiento científico. A mi parecer, tal procedimiento evidencia que la legislación arqueológica, al encargarse de las cosas, las reubica, a través de la asociación entre Arqueología empresarial y esa modalidad desvirtuada de Arqueología pública, en las redes de los discursos nacionalistas y colonialistas, aparte de asegurar los intereses del mercado y las políticas liberales.

En Brasil, según datos recientes, el 98\% de las autorizaciones emitidas por el Instituto do Patrimônio Histórico e Artístico Nacional (IPHAN) son destinadas a la Arqueología empresarial (Zanettini 2009; Wichers 2010; Tega 2012). En Argentina, así como en otros países suramericanos, la Arqueología empresarial se está posicionando firmemente en el escenario académico. La justificación para invasión empresarial, particularmente en Brasil, se asemeja al tercer eslogan del INRAP. En nuestro caso, disociada, para bien o para mal, de los usos nacionalistas. Pero, en estos momentos en que la economía brasileña ha crecido exponencialmente, la Arqueología empresarial, que se presenta ahora como "Arqueología pública", se apropia de las cosas arqueológicas y reproduce el colonialismo interno accionado por la Arqueología brasileña del siglo XIX y comienzos del XX (Ferreira 2010b): aliena las historias nativas $\mathrm{y}$ legitima la expropiación de las tierras.

Concluyo recordando lo que dijo David Lowenthal (2005) en un debate con Henry Merryman: las leyes patrimoniales raramente funcionan, pues la cultura material, además de estructurante, se cobra vida de acuerdo con los contextos y las cosmologías de las comunidades. Es verdad. Sin embargo, se debe tener en cuenta, en las investigaciones que hacemos sobre la capacidad de acción de la cultura material, que las legislaciones arqueológicas ayudan a encasillar las cosas; y hoy día, se convierten crecientemente en alegorías, emblemas de banderas nacionalistas y colonialistas, atracciones del turismo global y soportes del mercado liberal. 


\section{REFERENCIAS BIBLIOGRÁFICAS}

Andersen, B. (1983): Imagined Communities: Reflections on the Origin and the Spread of Nationalism. Verso, London.

Appadurai, A. (ed.) (1988): The Social Life of Things: Commodities in Cultural Perspective. Cambridge U. P, Cambridge.

Appadurai, A (2002): The Globalization of Archaeology and Heritage. Journal of Social Archaeology, 1: $35-49$.

Armstrong-Fumero, A. (2009): A Heritage of Ambiguity: the Historical Substrate of Vernacular Multiculturalism in Yucatán, México. American Ethnologist, 36 (2): 300-316.

Augé, M. (2007): Não-Lugares: Uma Introdução à Antropologia da Sobremodernidade. 90 Graus Editora, Lisboa.

Baram, U. (2007): Appropriating the Past: Heritage, Tourism, and Archaeology in Israel. Selective Remembrances: Archaeology in the Construction, Commemoration, and Consecration of National Pasts (P. Kohl, M. Kozelsky, N. Ben-Yuda, eds.). The University of Chicago Press, Chicago: 229-325.

Bate, F. (1998): La Investigación en Arqueología. Crítica, Barcelona.

Bauman, Z. (1988): Sobre o Comunitarismo e a Liberdade Humana, ou como Enquadrar o Círculo. O MalEstar da Pós-Modernidade. Jorge Zahar Editor, Rio de Janeiro: 231-245.

Belli, E; Slavutsky, R. (2010): A Gestão Estatal do Patrimônio para o Desenvolvimento dos Setores Polulares da Quebrada de Humahuaka. Patrimônio Cultural no Brasil e na Argentina: estudos de caso, (L. M. Ferreira, M. L. M. Ferreira, M. Rotiman, eds.). CAPES/Annablume, São Paulo: 145-159.

Bezerra, M. (2012): Signifying Heritage in Amazon: A Public Archaeology Project At Vila De Joanes, Marajó Island, Brazil. Chungara, 44: 533-542.

Bнавна, H. K. (1990): Dissemination. Nation and Narration. Routledge, London: 291-322.

Buchl, T. (2007): Material Culture: Current Problems. A Companion to Social Archaeology (L. Meskell, R. W. Preucel, eds.). Blackwell Publishing, Oxford: 179-194.

Burckhardt, J. (2012): O Estado como Obra de Arte. Penguin e Companhia das Letras, São Paulo.

CArman, J. (2005): Against Cultural Property: Archaeology, Heritage and Ownership. Duckworth, London.

Chambers, E. (2000): Native Tours: The Anthropology of Tourism and Travel. Prospect Heights, III: Waveland Press, London.

Colley, S. (2002): Uncovering Australia: Archaeology, Indigenous People and the Public. Allen and Unwin, Sidney.

Colwell-Chanthaphonh, C.; Ferguson, T.J. (ed.). (2008): Collaboration in Archaeological Practice: engaging descendent communities. Altamira Press, Lanham.

De Certeau, M. (1993): La Culture au Pluriel. Éditions du Seuil, Paris.

Deleuze, G; Guattari, F. (1980): Mille Plateaux: Capitalisme et Schizophrénie. Éditions de Minuit, Paris.

Elias, N. (1994): A Sociedade dos Individuos. Jorge Zahar Editor, Rio de Janeiro.

Eremites de Oliveira, J; Pereira, L. M. (2012): Terra Indígena Buriti: perícia antropológica, arqueológica e histórica sobre uma terra terena na serra de Macaraju, Mato Grosso do Sul. UFGD, Dourados.

FaulKner, N. (2000): Archaeology from Below. Public Archaeology, 1 (1): 21-33.

Ferreira, L. M. (2009a): Patrimônio Arqueológico, Pós-Colonialismo e Leis de Repatriação. Patrimônio Cultural e Ambiental: questões legais e conceituais. (P. P. Funari, S. Pelegrini, G. Rambelli, eds.). Annablume, São Paulo: 77-96.

Ferreira, L. M. (2009b): Arqueologia da Escravidão e Arqueologia Pública: Algumas Interfaces. Vestígios: Revista Latino-Americana de Arqueologia Histórica, 3 (1): 9-23.

Ferreira, L. M. (2010a): Arqueología Comunitaria, Arqueología Empresarial y Educación Patrimonial en Brasil. Jangwa Pana: Revista del Programa de Antropología de la Universidad del Magdalena, 9: 95-102.

Ferreira, L. M. (2010b). Território Primitivo: a institucionalização da arqueologia no Brasil (1870-1917). Editora da PUCRS, Porto Alegre. 
Ferreira, L. M; SAnches, P. L. M. (2011): Arqueologia de Contrato e Educação Patrimonial no Brasil: algumas provocações. Patrimônio Cultural no Brasil e na Argentina: estudos de caso, (L. M. Ferreira, M. L. Ferreira, M. Rotman, eds.). São Paulo: CAPES/Annablume, São Paulo: 161-172.

Foucault, M. (1996): ¿Qué es la Ilustración? Las Ediciones de La Piqueta, Madrid.

Foucault, M. (2002): A Verdade e as Formas Jurídicas. NAU Editora/PUC, Rio de Janeiro.

Foucault, M. (2008): O Nascimento da Biopolítica. Martins Fontes, São Paulo.

Fourmille, H. (1989). Who owns the past? Aborigines as captives of the archives. Aboriginal History, 13 (1): $1-8$.

Funari, P. P; Bezerra, M. (2012): Public Archaeology in Latin America. The Oxford Handbook of Public Archaeology, (R. Skeates, C. Macdavid, J. Carman, eds.). Oxford University Press, Oxford: Vol. I, 100-115.

Funari, P. P; Carvalho, A. V. (2012): Inclusion in Public Archeology in Brazil: Remarks on Collaborative Practices. Archaeologies, 8: 5-25.

Gillman, D. (2010): The Idea of Cultural Heritage. Cambridge U. P, Cambridge.

Given, M. (2004): The Archaeology of Colonized. Routledge, London.

Gnecco, C. (2009): Caminos de la Arqueología: de la Violencia Epistémica a la Relacionalidad. Arqueologia Amazônica: História e Identidades, (L. M. Ferreira, ed.). Boletim do Museu Paraense Emílio GoeldiCiências Humanas, 4 (1): 15-26.

Gombrich, E. H. (1992): Réflexions sur l'histoire de l'arte. Jacqueline Chambon, Nímes.

Gosden, C. (2001): Postcolonial Archaeology: Issues of Culture, Identity, and Knowledge. Archaeological Theory Today, (I. Hodder, ed.). Cambridge: Polity Press, Cambridge: 242-261.

Gosden, C. (2004): Archaeology and Colonialism: Cultural Contact from 5000 BC to the Present. Cambridge U. P, Cambridge.

Gosden, C. (2005): What do Objects Want? Journal of Archaeological Method and Theory, 12 (3): 93-211.

Guthrie, T. H. (2010): Dealing with Difference: Heritage, Commensurability and Public Formation in Northern New Mexico. International Journal of Heritage Studies, 16: 305-321.

Hamilakis, Y; Anagnostopoulos, A. (2009): Public archaeology: archaeological ethnographies. Public Archaeology, 8: 65-87.

HodDer, I. (2012): Entangled: An Archaeology of the Relationships between Humans and Things. WileyBlackwell, Oxford.

Holtorf, Cornelius. (2006): Can less be more? Heritage in the Age of Terrorism. Public Archaeology, 5: 101-110.

Horkheimer, M. (1970): Origens da Filosofia Burguesa da História. Editorial Presença, Lisboa.

Hume, I. N. (1969): The Why, What, and Who of Historical Archaeology. Historical Archaeology: A Comprehensive Guide for Both Amateurs and Professionals to the Techniques and Methods of Excavating Historical Sites. Alfred Knopf, New York: 7-20.

Jones, A. (2007): Memory and Material Culture. Cambridge U. P, Cambridge.

Kohl, P; Kozelsky, M; Ben-Yehuda, N. (2007): Introduction: Selective Remembrances: Archaeology in the Construction, Commemoration, and Consecration of National Pasts. Selective Remembrances: Archaeology in the Construction, Commemoration, and Consecration of National Pasts, (P. Kohl, M. Kozelsky, N. Ben-Yehuda, eds.). The University of Chicago Press, Chicago: 1-28.

Latour, B. (2008): Reensamblar lo Social: una introducción a la teoría del actor-red. Manantial, Buenos Aires.

Little, B. (ed). (2002): Public Benefits of Archaeology. University Press of Florida, Florida.

Lowenthal, D. (2005): Why Sanctions Seldom Work: Reflections on Cultural Property Nationalism. International Journal of Cultural Property, 12: 393-423.

Marshall, Y. (2002): What is Community Archaeology? World Archaeology, 32 (2): 211-219.

Macdavid, C. (2004): From "Traditional” Archaeology to Public Archaeology to Community Archaeology. Places in Mind: Public Archaeology as Applied Anthropology, (P. A. Shackel. E. J. Chambers, eds.). Routledge, London: 15-35 
Mcgimsey, Ch. (1972): Public archaeology. Seminar Press, New York.

Merriman, N. (ed.). (2004): Public Archaeology. Routledge, London.

Merryman, J. H. (2000): Thinking about the Elgin Marbles: Critical Essays on Cultural Property, Art and Law. Kluwer Law International, London.

Meskell, L. (ed.). (1998): Archaeology under Fire: nationalism, politics and heritage in the Eastern Mediterranean and Middle Eastern. Routledge, London.

Meskell, L. (2002): Negative Heritage and Past Mastering in Archaeology. Anthropological Quarterly, 75 (3): 557-574.

Olsen, B. (2010): In defense of things. Archaeology and the Ontology of Objects. AltaMira, Lanham, Maryland.

Rivolta, M. C; Montenegro, M; Argañaraz, C. del V. (2011): Multivocalidade nos Processos de Apropriação e Significação no Pucará de Tilcara. Patrimônio Cultural no Brasil e na Argentina: estudos de caso (L. M. Ferreira, M. L. M. Ferreira, M. Rotman, eds.). CAPES/Annablume, São Paulo: 173-192.

Sartre, Jean-Paul. (1979). Prefácio. Os Condenados da Terra (F. Fanon). Civilização Brasileira, Rio de Janeiro: 3-21.

Schofield, J; Johnson, W. G. (2006): Archaeology, Heritage and the Recent and Contemporary Past. Historical Archaeology (D. Hicks, M.C. Beaudry, eds.). Cambridge U. P., Cambridge: 104-122.

Shackel, P. (2001): Public Memory and the Search for Power in American Historical Archaeology. American Anthropologist, 103 (3): 655-670.

Shepherd, N. (2009): “Cuando la Mano que Sostiene el Palustre es Negra...”. Prácticas Disciplinarias de Auto-Representación y el Asunto de la Mano de Obra "Nativa" en Arqueología. Arqueología Suramericana, 5 (1): 3-20.

Silva, F. A. (2011): Patrimônio Arqueológico em Terras Indígenas: Considerações sobre o Tema no Brasil. Patrimônio Cultural no Brasil e na Argentina: estudos de caso (L. M. Ferreira, M. L. M. Ferreira, M. Rotman, eds.). CAPES/Annablume, São Paulo: 193-218.

Silverman, H. (2002): Touring Ancient Times: The Present and Presented Past in Contemporary Peru. American Anthropologist, 104 (3): 881-902.

Simpson, M. G. (2001): Making Representations: Museums in the Post-Colonial Era. Routledge, London.

Simpson, F; Williams, H. (2008): Evaluating Community Archaeology in the UK. Public Archaeology, 7 (2): 69-90.

Skinner, Q. (1996): As Fundações do Pensamento Político Moderno. Companhia das Letras, São Paulo.

Sмith, C; Wobst, H. M. (eds.). (2005): Indigenous Archaeologies: Decolonizing Theory and Practice. Routledge, London.

Tega, G. (2012): Arqueologia no Brasil e o Panorama Atual: os números de 11 anos de divulgação na Folha de São Paulo. Arqueologia Pública, 5: 14-27.

Thomas, N. (1991): Colonialism's Culture: Anthropology, Travel, and Government. Princeton U. P., Princeton

Trigger, B. G. (1984): Alternative Archaeologies: Nationalist, Colonialist, Imperialist. Man, 19: 355-370.

Tully, G. (2007): Community Archaeology: General Methods and Standards of Practice. Public Archaeo$\log y, 6: 155-187$.

Turner, V. (2005): A Floresta dos Símbolos: Aspectos do Ritual Ndembu. Editora da UFF, Niteroi.

Watson, P. J. (2008): Processualism and After. Handbook of Archaeological Theories (R. A. Bentley, H. D. G. Maschner, C. Chippindale, eds.). Altarmira Press, New York: 29-37.

Wichers, C. A. DE Moraes. (2010): Museus e Antropofagia do Patrimônio Arqueológico: caminhos da prática brasileira. Tese de Doutorado, Programa de Pós-graduação em Museologia, Universidade Lusófona de Humanidades e Tecnologia, Lisboa.

Zanettini, P. E. (2009): Projetar o Futuro para a Arqueologia Brasileira: um desafio de todos. Revista Americana, 27: 71-87. 